\title{
Real-time control of polarisation in ultra-short-pulse laser micro-machining
}

\author{
O.J. Allegre · W. Perrie · K. Bauchert • D. Liu • \\ S.P. Edwardson • G. Dearden • K.G. Watkins
}

Received: 28 March 2011 / Accepted: 19 December 2011

(c) Springer-Verlag 2012

\begin{abstract}
The use of a fast-response, transmissive, ferroelectric liquid-crystal device for real-time control of the polarisation direction of a femtosecond laser beam, and the benefits for various aspects of ultra-short pulse micromachining, are discussed. Several configurations have been used to drive the polarisation in real-time. Our microscopic investigations of the resulting features revealed significant improvements in process efficiency and quality, compared to static linear and circular polarisations. Following our successful micro-machining tests, real-time polarisation control could emerge as a powerful tool in laser engineering.
\end{abstract}

\section{Introduction}

The past decade has seen significant developments in ultrashort pulse laser micro-processing. Thanks to the ultra-short timescale on which laser energy is coupled to the material, sub-micron precision processing can be achieved with very little thermal damage [1-3]. As a result, processes based on femtosecond and picosecond laser pulse durations have become increasingly widespread. Industrial applications include the very precise drilling of holes for fuel-injection nozzles [3], the processing of silicon wafers [4], or the precise machining of medical stent devices [5, 6].

O.J. Allegre $(\bowtie) \cdot$ W. Perrie $\cdot$ D. Liu $\cdot$ S.P. Edwardson

G. Dearden · K.G. Watkins

Laser Group, School of Engineering, University of Liverpool,

L69 3GQ, Liverpool, UK

e-mail: O.Allegre@liv.ac.uk

Fax: +44-(0)151-7944585

K. Bauchert

Boulder Nonlinear Systems, Inc., Lafayette, CO 80026, USA
The key parameters influencing the laser process quality and efficiency are the fluence, pulse duration, wavelength, beam quality and polarisation [7-9]. The influence of polarisation on micro-machining has been experimentally demonstrated in [9-12], showing that drilling high-aspectratio (depth/diameter) microscopic holes in metal with linear polarised ultra-short-pulse lasers produces anisotropic profiles. This is due to the higher reflectivity of the $s$-polarised radiation, relative to the $p$-polarised radiation [10]. As the hole develops through the material, the $p$-polarised radiation is more readily absorbed in the sidewalls, whereas the $s$-polarised radiation tends to be reflected down to the base of the hole. This results in a distorted intensity profile, affecting the shape of the hole when the beam reaches the exit side. The simplest way to reduce these distortions is to use a circular polarised beam, which removes the differential in reflectivity during drilling. In some cases, however, the remaining distortions associated with circular polarisation are problematic. In particular, ripple formation on the surface of the side wall cannot be avoided [9]. Another aspect of importance for micro-machining is the laser process efficiency. The influence of polarisation on this has been studied in $[13,14]$. When drilling or cutting high-aspect-ratio microscopic features, neither circular nor static linear polarisation offers the optimum process efficiency.

To overcome these limitations, another technique referred to as polarisation trepanning was developed [10]. It consists of rotating linear polarisation during drilling, further improving the hole quality $[10,12,15]$. The trepanning optic developed in [11] produced holes of remarkable quality using this technique. However, these methods involve mechanical rotation of optical components and could be adversely affected by vibrations and prone to mechanical failure, leading to potentially expensive maintenance. An alternative laser-specific polarisation switching method 
is detailed in [8]. It is based on an intra-cavity polarisation chopper wheel synchronised to the laser pulse train. However, as this method requires the laser cavity to be redesigned, it cannot be easily applied to existing laser systems.

In this paper, we propose a flexible method for rapidly switching the linear polarisation of a laser beam between two orthogonal directions during micro-processing, using a fast-response liquid-crystal polarisation rotator. This novel method aims to provide an alternative to those mentioned in the previous paragraph, resolving the issues related to linear polarisation (poor process quality), circular polarisation (ripple formation and poor process efficiency) and polarisation trepanning with its reliance on mechanical rotation stages. As a proof of principle, helical drilling and cutting tests were performed on stainless steel sheets using various polarisation configurations. Experimental results using a femtosecond laser show a consistent improvement in the micro-processing quality compared to that produced by linear polarisation configurations.

\section{Experimental setup}

A schematic of the experimental setup is shown in Fig. 1. The output from a femtosecond laser (Clark-MXR CPA2010, with a minimum pulse width of $160 \mathrm{fs}, 775 \mathrm{~nm}$ central wavelength, spectral bandwidth of $5 \mathrm{~nm}, 1 \mathrm{~mJ}$ maximum pulse energy, $1 \mathrm{kHz}$ repetition rate and horizontal linear polarisation) is attenuated by a half-wave-plate and a Glan laser polariser. The resulting linear polarised beam is incident on a transmissive, ferroelectric liquid-crystal polarisation rotator developed by Boulder Nonlinear Systems,
Inc. (MS Series). The liquid-crystal polarisation rotator offers a switching frequency of up to $20 \mathrm{kHz}$ between two orthogonal output states, with a response time of typically less than $100 \mu \mathrm{s}$. Varying the electric field applied to the device results in a non-linear rotation of the output polarisation. The liquid-crystal device can be operated in a binary mode, where the polarisation is periodically flipped between orthogonal directions, or operated in a quasi-linear mode. The polarisation rotator is driven by a programmable, DC-balanced, periodic voltage signal from a function generator (Tektronix AFG3021B). After reflection on Mirrors 3 and 4 (see Fig. 1), the beam enters the $10 \mathrm{~mm}$ aperture of a scanning galvo system (GSI Lumonics) with a flat field lens $(f=100 \mathrm{~mm})$, driven by a programmable PC interface board (Scanlab). Samples are mounted on a precision 4-axis $(x, y, z, \theta)$ motion control system (Aerotech Npaq) allowing accurate positioning of the sample at the laser focus.

\section{Initial processing results and discussion}

\subsection{Testing of response time}

The response time of the liquid-crystal device was tested by placing a Glan laser polariser behind it. Thus, a rotation in polarisation direction was translated into a modulation of the amplitude transmitted through the polariser. The resulting beam amplitude was measured with a photodiode (see dashed components in Fig. 1). In order to obtain a clearer signal from the photodiode, the polarisation rotator driving signal was synchronised with the $1 \mathrm{kHz}$ femtosecond laser pulse train. This was achieved by triggering the function generator with a synchronised TTL signal from the

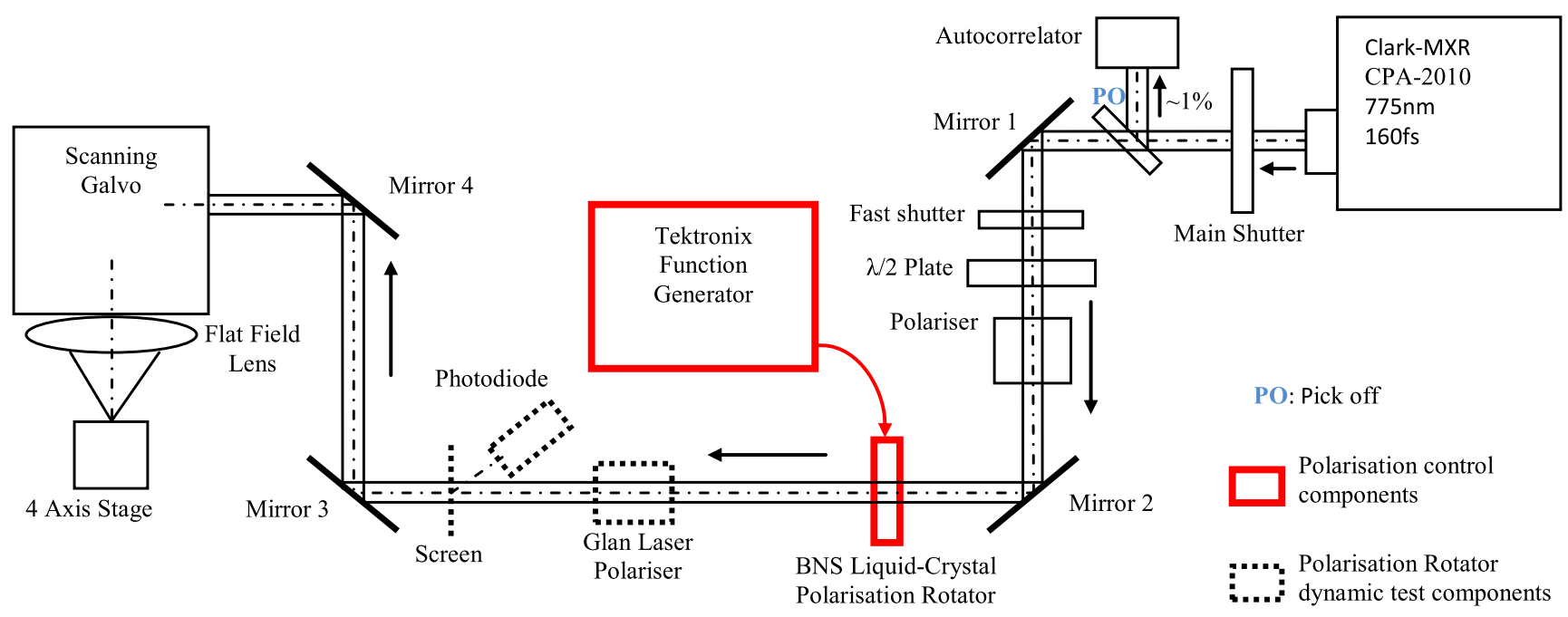

Fig. 1 Micro-machining experimental setup showing how the liquid-crystal polarisation rotator was used. For the processing tests, the dashed components were removed. This setup is a modified version of the one used in [16] 


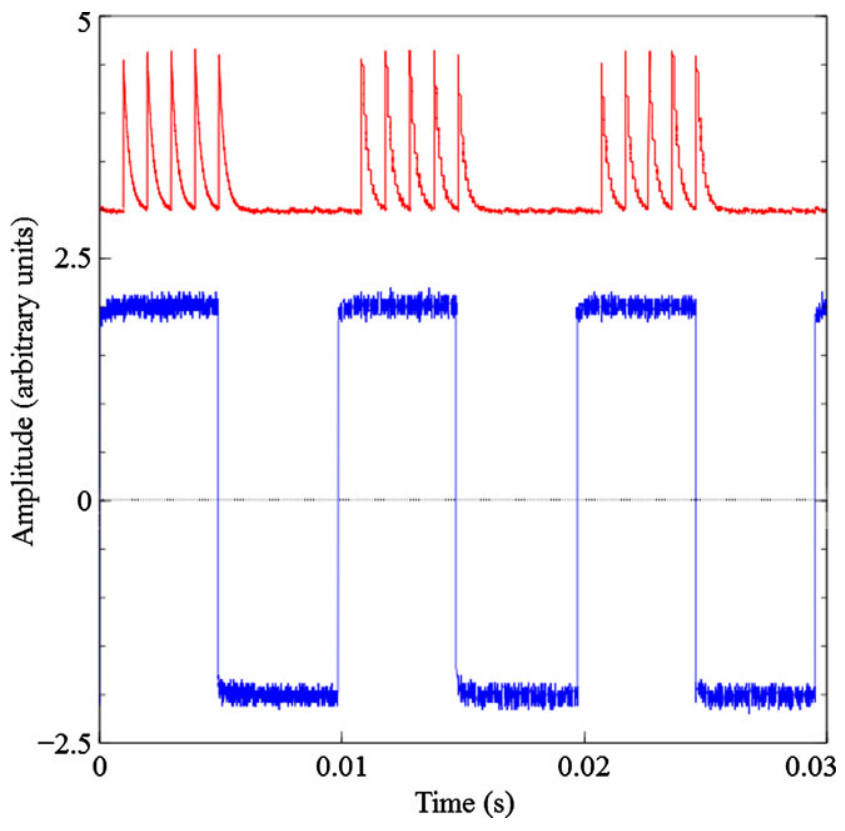

Fig. 2 Oscilloscope traces showing the amplitude of the femtosecond pulse train (output signal from the photodiode in red on top) modulated by a polarisation rotator with a Glan laser polariser behind it (driving signal in blue at the bottom)

femtosecond laser's Pockels cell driver (DT-505). A DCbalanced, $4 \mathrm{~V}$ peak-to-peak square-wave signal was programmed on the function generator, triggered by this synchronised signal. Oscilloscope traces from the photodiode and the driving signal of the liquid-crystal device are shown in Fig. 2. When the driving voltage is positive $(+2 \mathrm{~V})$, the resulting polarisation is parallel to the transmission axis of the Glan laser polariser. The transmitted laser pulse train measured by the photodiode shows a residual amplitude shift due to the inertia of the liquid-crystal device. When the driving voltage is negative $(-2 \mathrm{~V})$, the laser pulse train is blocked by the polariser. The response time of the polarisation rotator was found to be less than $0.5 \mathrm{~ms}$ (manufacturing specification: $0.1 \mathrm{~ms}$ ). In the Clark laser system, the period between each femtosecond pulse is $\sim 1 \mathrm{~ms}$ ( $1 \mathrm{kHz}$ repetition rate). The response time of the liquid-crystal device is therefore sufficient to provide accurate control over the polarisation direction of each individual pulse. When a polarising filter is placed behind the liquid-crystal rotator, this pulse-train-synchronised mode enables dynamic pulse-topulse amplitude control, with potential applications in surface machining of periodic structures.

\subsection{Polarisation diagnostic}

Periodic surface structures produced by laser irradiation of solid material close to the ablation threshold have been widely observed since the early 1970s. They are referred to as Laser Induced Periodic Surface Structures (LIPSS). Details of their properties can be found in [17-20]. When a steel work piece is exposed to a laser beam with an intensity close to its ablation threshold, periodic surface ripples develop in a direction perpendicular to the linear polarisation. Thanks to their diffractive properties, these ripples provide a marker for the direction of polarisation of the incoming laser beam.

The aim of the polarisation diagnostic tests was to demonstrate that polarisation trepanning was achievable with the liquid-crystal device. Polarisation trepanning requires synchronising the polarisation rotation with the beam scanning motion so that the direction of polarisation is constantly perpendicular to the direction of scanning [9]. This is normally achieved by placing a wave-plate in the optical path and mechanically rotating it with the beam. In order to obtain a similar effect, the driving signal from the function generator was synchronised with the galvo scanning head (see Fig. 1). A test procedure was developed to visualise the direction of polarisation along the scanned beam paths by making use of LIPSS. We used the base setup shown in Fig. 1 (without the dashed components).

Due to its design characteristics, the response time of the liquid-crystal device is shorter when the polarisation is flipped between two perpendicular directions and longer when it is gradually rotated. The former case applies a DCbalanced square-wave driving signal, the latter applies a sinusoidal one. In the following test, a square-wave signal was used to obtain the best polarisation switching speeds. Synchronisation routines were programmed into the PC interface board driving the galvo head to maintain the desired polarisation state while scanning along pre-defined geometries on a steel sample surface (Fig. 3a). The scanning programmes were run with the laser pulse energy attenuated to $2 \mu \mathrm{J}$ and the sample was exposed to the laser beam for a few seconds. As a result, LIPSS were produced and later imaged with an optical microscope (Fig. 3b).

The microscopic investigation of the resulting figures used low-angle illumination to distinguish between the two directions of laser polarisation that produced the LIPSS. Two illumination sources were used, located on each side of the sample, with the axis of illumination set parallel to one of the polarisation directions and perpendicular to the other. Due to the diffractive properties of the LIPSS, only the direction of polarisation parallel to the direction of illumination reflected the low-angle light (see Fig. 3c), the other remained dark. Therefore, each microscope image shows the part of the scanned geometry that reflected the illumination. Rotating the axis of illumination by $90^{\circ}$ reveals the remainder of the scanned geometry (Fig. 3d). These tests demonstrated that the desired direction of polarisation had been successfully applied to each region, giving confidence that a good approximation of polarisation trepanning is achievable using a liquid-crystal polarisation rotator. 


\section{Helical drilling test results and discussion}

In helical drilling, the laser beam moves in a periodic fashion following a pre-defined geometry. As material is removed by each pulse, the beam works its way through the sam-

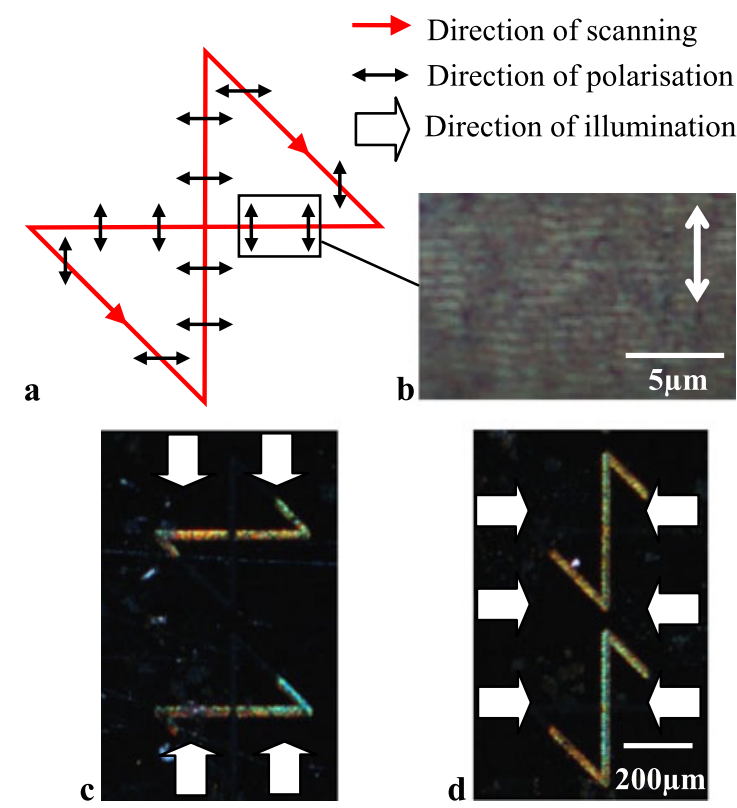

Fig. 3 (a) Programmed laser beam path showing the expected state of polarisation for each region. (b) LIPSS formation on the surface of the work piece. The direction of polarisation is indicated by the white arrows. (c) Optical micrograph of the scanned beam path after laser exposure, under low-angle illumination. (d) The same region after the illumination was rotated by $90^{\circ}$, revealing the remainder of the scanned geometry ple (in the case of a circular beam motion, the beam front follows a helical path). The parameters influencing the geometry of the machined hole include the dimensions of the programmed beam path, the laser spot size and the polarisation [9]. To check the benefits of using a liquid-crystal polarisation rotation device in helical drilling, tests were performed on $380 \mu \mathrm{m}$-thick stainless steel samples in air, using circular and square beam paths.

\subsection{Circular beam path}

Circular beam paths with diameters of $65 \mu \mathrm{m}$ were programmed into the PC interface board driving the scanning galvo. A laser pulse energy of $75 \mu \mathrm{J}$ was used, corresponding to a peak fluence of $\sim 24 \mathrm{~J} / \mathrm{cm}^{2}$, much higher than the ablation threshold which is around $0.16 \mathrm{~J} / \mathrm{cm}^{2}$ for stainless steel [21]. The spot size (calculated) is $30 \mu \mathrm{m}$. The chosen drilling time per hole was $150 \mathrm{~s}$. The test strategy consisted of using the various polarisation modes available with the liquid-crystal polarisation rotator, varying the operating parameters to improve circularity and reduce the taper of the micro-holes. Linear and circular polarised beams were used to provide comparative data for subsequent tests. The experimental configuration of the setup for each test is summarised in Table 1.

All these tests produced tapered holes with an entrance opening diameter of typically $110 \pm 10 \mu \mathrm{m}$. The entrances of the holes showed no dependence on polarisation, but were slightly elliptical in shape due to the slight astigmatism of the incident laser beam profile (Fig. 4). On the exit side, the shape and taper of the holes varied with polarisation, with

Table 1 Description and results of helical drilling tests on a $380 \mu \mathrm{m}$-thick stainless steel plate, including a comparison of the exit holes surface area and ellipticity $(775 \mathrm{~nm}, 160 \mathrm{fs}$ pulses, $1 \mathrm{kHz}$ repetition rate, pulse energy: $75 \mu \mathrm{J})$

Linear polarisation

Circular polarisation

Scanner-
synchronised
polarisation
switching

Configuration of the setup

Optical micrographs of the holes exit aperture (arrows indicate the direction of polarisation)

Ellipticity
Surface area
Polarisation rotator removed. The linear polarised laser beam is used to drill the sample.

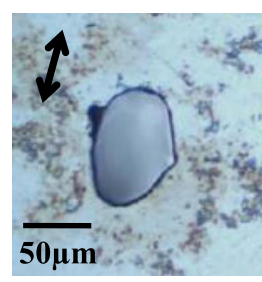

$$
35 \pm 5 \%
$$$$
3000 \pm 300 \mu \mathrm{m}^{2}
$$

Polarisation rotator removed.

A quarter-wave plate is used to produce circular polarisation.

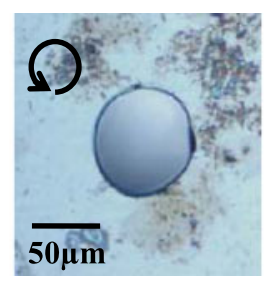

$10 \pm 5 \%$

$3400 \pm 300 \mu \mathrm{m}^{2}$
Polarisation rotator driven with a $10 \mathrm{~Hz}$ square wave synchronised with the galvo scanner.

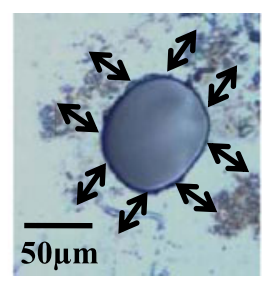

$15 \pm 5 \%$

$3700 \pm 300 \mu \mathrm{m}^{2}$
Unsynchronised

polarisation

switching
Polarisation-modulated geometry

Polarisation rotator driven with an unsynchronised $10 \mathrm{~Hz}$ square wave.
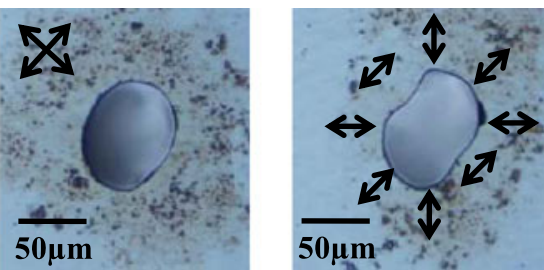

Polarisation rotator driven with a $10 \mathrm{~Hz}$ sine wave synchronised with the galvo scanner.

\section{$50 \mu \mathrm{m}$}

N/A

$3300 \pm 300 \mu \mathrm{m}^{2}$ 


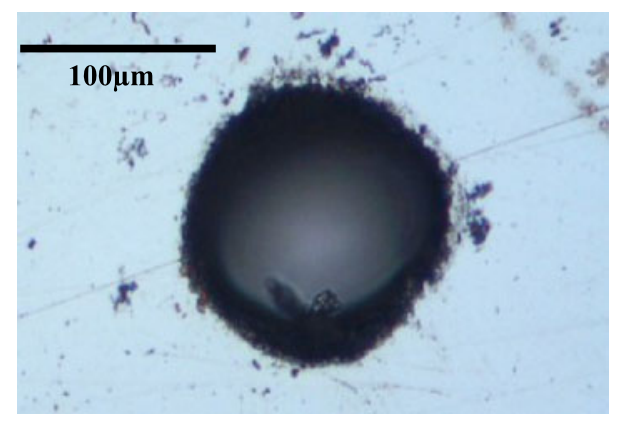

Fig. 4 Optical micrograph of the hole entrance. The shape of the hole does not depend on polarisation

a typical half-angle sidewall taper ranging between $4^{\circ}$ and $5^{\circ}$ and a diameter of typically $65 \pm 10 \mu \mathrm{m}$. A summary of the test results as well as optical micrographs of the exit holes for the various polarisation modes are given in Table 1 .

\subsubsection{Linear and circular polarisations}

Drilling with a linearly polarised laser beam produces elliptical exit holes, elongated in the direction of the polarisation vector (see Table 1). As the laser beam reflects against the internal sidewalls during drilling, the light is $p$-polarised in the regions where the sidewalls are perpendicular to the polarisation vector (i.e. where the plane of incidence is parallel to the polarisation vector) and $s$-polarised in the regions where the sidewalls are parallel to it (i.e. where the plane of incidence is perpendicular to the polarisation vector). The laser energy is more readily absorbed in the regions with $p$-polarisation, producing more ablation which results in an elliptical profile with a long axis parallel to the polarisation vector. $s$-polarisation tends to be reflected down to the base of the hole. As the sidewalls are not optically flat, these internal reflections sometimes produce distortions around the exit hole, perpendicular to the polarisation vector as can be seen on the hole drilled with a linearly polarised beam in Table 1. As expected, circular polarisation produced a clear, circular hole exit due to the isotropic coupling of the beam energy to the material in this case.

\subsubsection{Scanner-synchronised polarisation switching}

In polarisation trepanning, the direction of linear polarisation rotates synchronously with the beam around the hole so that it is always oriented in the same way with regard to the wall [9]. To obtain a similar effect, our galvo scanner was synchronised with the liquid-crystal polarisation rotator, driven with a $4 \mathrm{~V}$ peak-to-peak square-wave. This test produced an approximation of polarisation trepanning by maintaining the direction of polarisation as close as possible to a $p$-polarisation during helical drilling (see Fig. 5). The

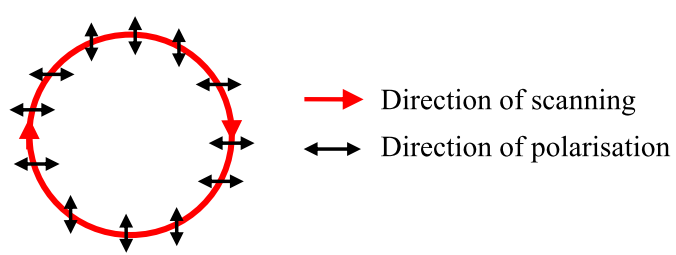

Fig. 5 Laser scanning path with corresponding direction of polarisation used in polarisation trepanning

experimental results obtained are described in Table 1. It can be seen that the exit apertures of the holes drilled using this method have an increased surface area $(\sim 10 \%)$ compared to circular polarisation, demonstrating an improved coupling of the laser energy to the stainless steel and an increase in the machining efficiency. However, the quality is limited by only having two directions of polarisation; it is more promising for drilling geometrical shapes with straight edges, such as those shown in Table 2.

\subsubsection{Un-synchronised polarisation switching}

In these tests, the square-wave signal driving the polarisation rotator was not synchronised with the beam motion. During helical drilling, the laser beam rotates about the central axis of the hole. When the polarisation rotator is not synchronised with the scanner system, a given location in the circular beam path will see the polarisation direction vary over time. The resulting reflectivity of the laser beam at this location will vary accordingly. This is known to lead to an averaging effect which tends to reduce the distortions in the exit hole [10]. As expected, our un-synchronised helical drilling tests showed reduced distortions compared to the other polarisation modes (see Table 1). It is noted that the slight increase in ellipticity is due to the rotation span of the liquidcrystal device, which is limited to $90^{\circ}$.

\subsubsection{Polarisation modulated geometry}

In the drilling tests described above, real-time polarisation control was used to improve the circularity of the exit holes. However, some applications require drilling non-circular holes [9]. Consequently, we investigated whether polarisation control could be used for drilling pre-defined noncircular holes, using the same circular beam path as in the previous tests. Instead of using a square-wave signal as before, we used a sinusoidal one to drive the polarisation rotator. This results in a gradual rotation of polarisation between orthogonal directions, instead binary switching as is the case when a square-wave signal is used. The same synchronisation setup as in polarisation trepanning was used and the other laser drilling parameters were similar. These tests produced a repeatable exit hole geometry. This geometry (see Table 1) did not result from the (circular) beam scanning 
Table 2 Description and results of square-shaped helical drilling tests on a $380 \mu \mathrm{m}$-thick stainless steel plate $(775 \mathrm{~nm}, 160 \mathrm{fs}$ pulses, $1 \mathrm{kHz}$ repetition rate, pulse energy: $75 \mu \mathrm{J}$ )

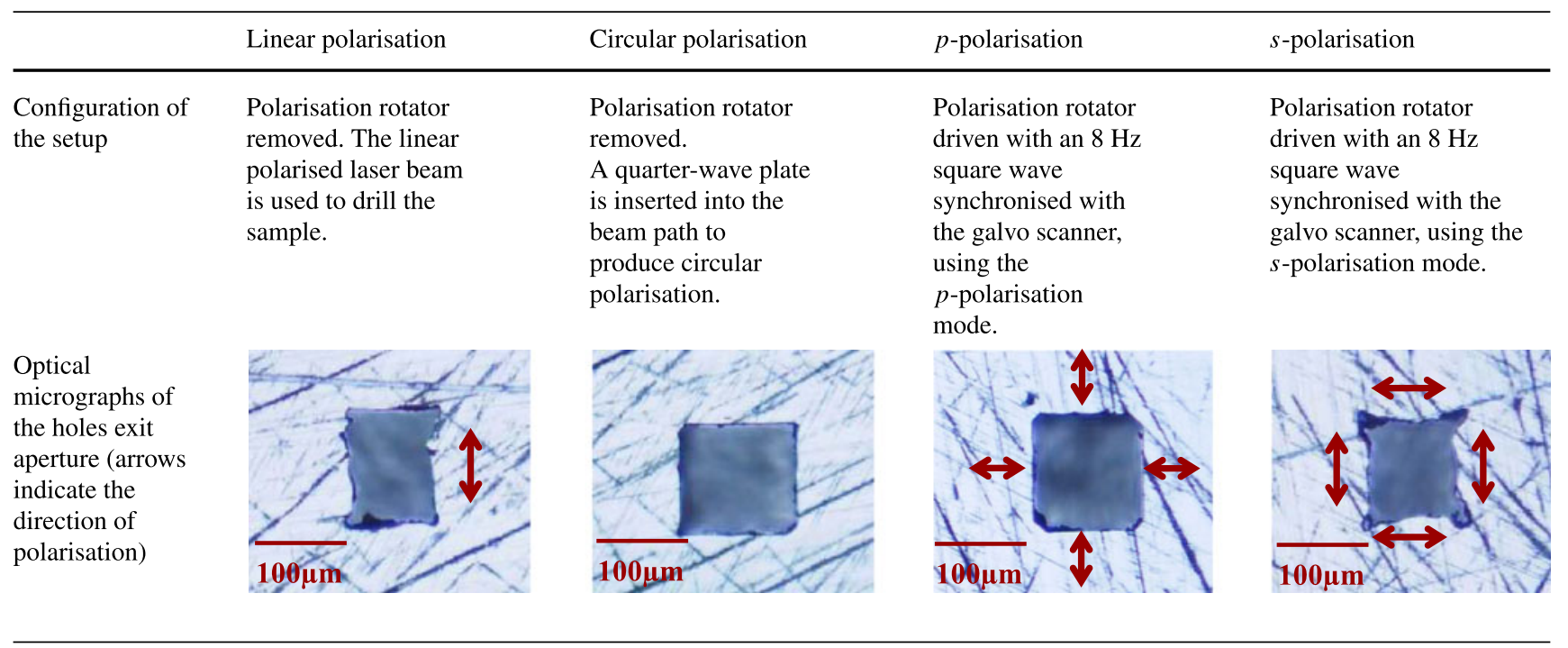

path but from the modulation of polarisation. This technique could provide more flexibility in micro-drilling non-circular holes.

\subsection{Square beam path}

The limitation on the available rotation span $\left(90^{\circ}\right.$ with this device) means that the quality of straight orthogonal edged geometries is likely to be most improved. To confirm this prediction, we drilled and analysed square holes. The liquidcrystal device was configured in scanner-synchronised polarisation switching mode (using a $10 \mathrm{~V}$ peak-to-peak square-wave driving signal) so that polarisation trepanning could be achieved. Both $p$ - and $s$-polarisations were tested i.e. the polarisation direction during drilling was always maintained at $p$ - or $s-$, respectively. Prior to drilling, a synchronisation program was developed and tested by producing LIPSS on a sample, using the polarisation diagnostic procedure described earlier.

The programmed square beam paths had a side length of $130 \mu \mathrm{m}$. For the drilling tests, a laser pulse energy of $75 \mu \mathrm{J}$ was used $\left(F \sim 24 \mathrm{~J} / \mathrm{cm}^{2}\right)$. This produced square holes with an entrance side length of $\sim 200 \mu \mathrm{m}$ and an exit side length of $\sim 120 \mu \mathrm{m}$, corresponding to a $\sim 6^{\circ}$ half-angle sidewall taper. The entrance of the square holes showed no dependence on polarisation (Fig. 6). The distortions in the corners at the entrance were due to the limited resolution of the scanning galvo. The exit holes obtained are shown in Table 2. It is noted that $s$-polarisation produces the worst machining quality, with distorted exit holes. This is due to the relatively poor coupling of the laser beam energy to the sidewalls. A larger share of the beam energy is reflected away through the exit aperture of the hole compared with

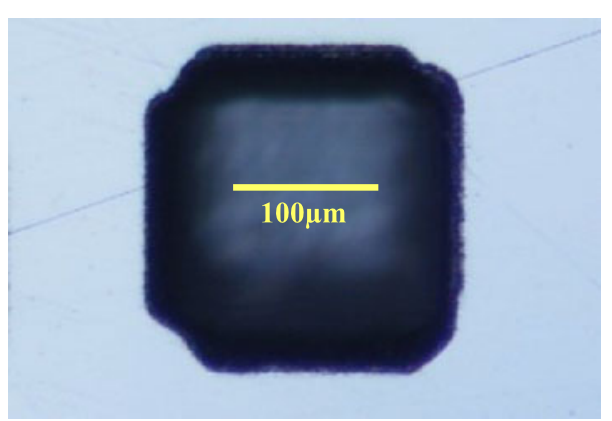

Fig. 6 Optical micrograph of the hole entrance. The shape of the hole does not depend on polarisation

$p$-polarisation, where the coupling to the sidewalls is better. As the $p$-polarisation achieved similar drilling quality as circular polarisation, these results suggest that our polarisation trepanning technique could be a viable alternative to static linear or circular polarisation.

\section{Cutting test results and discussion}

In the helical drilling tests described above, the chosen dimension for the holes was such that the resulting figure dimensions were not significantly larger than the laser spot size ( $\sim 120 \mu \mathrm{m}$ and $\sim 30 \mu \mathrm{m}$, respectively in Sect. 4.2). The relatively small hole dimension to spot size ratio $(\sim 4$ here $)$ means that all the material within the scanned figure is ablated. As a result, there is a continuous ablation front across the hole and this ablation front is wider than the laser spot itself. As material is removed by each laser pulse, the ablation front penetrates deeper inside the thickness of the material while maintaining an entrance opening that is wider 
Table 3 Description and results of cross-shaped cutting tests on a $380 \mu \mathrm{m}$-thick stainless steel plate (775 $\mathrm{nm}, 160 \mathrm{fs}$ pulses, $1 \mathrm{kHz}$ repetition rate, pulse energy: $75 \mu \mathrm{J})$

\begin{tabular}{|c|c|c|c|}
\hline & Linear polarisation & $p$-polarisation & $s$-polarisation \\
\hline $\begin{array}{l}\text { Polarisation } \\
\text { configuration during } \\
\text { machining }\end{array}$ & $\begin{array}{l}\text { The polarisation direction } \\
\text { (yellow arrow) is } p \text {-for the } \\
\text { horizontal branch and } s \text { - for } \\
\text { the vertical one. }\end{array}$ & $\begin{array}{l}\text { The polarisation direction } \\
\text { was maintained at } p \text { - (see } \\
\text { yellow arrows). }\end{array}$ & $\begin{array}{l}\text { The polarisation direction was } \\
\text { maintained at } s \text { - (see yellow } \\
\text { arrows). }\end{array}$ \\
\hline $\begin{array}{l}\text { Optical micrographs of } \\
\text { the holes exit aperture } \\
\text { (arrows indicate the } \\
\text { direction of polarisatio }\end{array}$ & & & \\
\hline
\end{tabular}

than the spot size. This is essential in maintaining a high processing efficiency while drilling high-aspect-ratio holes, as it enables effective channelling of laser energy inside the hole by avoiding excessive reflections on the walls. It also facilitates ejection of the ablated material outside of the hole.

In the case of micro-cutting, where the scanned figure is much larger than the laser spot size, only the material along the beam path is ablated. This means that high-aspectratio grooves are produced (see Table 3 ). As the ablation front is of a similar size to the laser spot, micro-cutting can suffer from poor process efficiency: multiple reflections on the groove walls lead to poor channelling of laser energy to the bottom of the groove. As the laser beam's polarisation can have a strong effect on these internal reflections, we investigated whether polarisation control is beneficial in improving laser micro-cutting efficiency and quality. The tests described below were performed on $380 \mu \mathrm{m}$-thick stainless steel samples.

\subsection{Cross-shaped beam path}

A cross-shaped geometry was used, which is equivalent to cutting two orthogonal linear slots. As in the square drilling tests described earlier, the liquid-crystal device was configured in scanner-synchronised polarisation switching mode, using a $10 \mathrm{~V}$ peak-to-peak square-wave driving signal to achieve polarisation trepanning. As before, both $p$ - and $s$ polarisation modes were used as well as a static linear polarisation for comparison purposes. A synchronisation program was developed and tested at low pulse energies $(2 \mu \mathrm{J})$ to produce LIPSS on the sample (see Fig. 3).

The micro-cutting tests used pulse energies of $75 \mu \mathrm{J}$ $\left(F \sim 24 \mathrm{~J} / \mathrm{cm}^{2}\right)$. The length of each slot was $450 \mu \mathrm{m}$.
The exit side of the resulting incisions can be seen in Table 3. Static linear polarisation produced varying degrees of anisotropic machining features. Visual inspection showed the regions of the beam path with $p$-polarised internal reflections were of much better quality (i.e. less distorted and with straighter edges) than the $s$-polarised regions. Our synchronisation program, which ensured that $p$-polarisation is used on both arms of the cross, improved both the straightness and the accuracy of the machined slots by minimising internal reflections and maintaining an optimum coupling of the laser beam energy to the sidewalls. It is noted that the bulge-shaped distortions at the end of each arm (see optical micrographs in Table 3) were not caused by polarisation, but rather resulted from the beam path geometry and the deceleration at the ends due to the inertia of the galvo mirrors (see Fig. 3 for beam path).

\subsection{Square beam path}

To further investigate the gains in cutting efficiency and quality, square holes were produced by cutting microgrooves through the stainless steel plates and removing the remaining square cut-out in the centre after laser exposure. As in the previous tests, the laser pulse energy was set at $75 \mu \mathrm{J}\left(F \sim 24 \mathrm{~J} / \mathrm{cm}^{2}\right)$. The side length of the scanned figures was $650 \mu \mathrm{m}$. The same polarisation trepanning technique was used as before. Both $p$ - and $s$-polarisation modes were tested as well as circular polarisation for comparison purposes. The chosen processing time per hole was $500 \mathrm{~s}$. After laser processing, the samples were placed in an ultrasonic bath for three minutes, so that the pressure waves would remove the central squares if the cutting process had been successful. The exit side of the resulting holes are shown in Table 4. 
Table 4 Description and results of square-shaped cutting tests on a $380 \mu \mathrm{m}$-thick stainless steel plate (775 nm, $160 \mathrm{fs}$ pulses, $1 \mathrm{kHz}$ repetition rate, pulse energy: $75 \mu \mathrm{J}$ )

\begin{tabular}{|c|c|c|c|}
\hline & Circular polarisation & $p$-polarisation & $s$-polarisation \\
\hline $\begin{array}{l}\text { Polarisation } \\
\text { configuration } \\
\text { during } \\
\text { machining }\end{array}$ & $\begin{array}{l}\text { A quarter-wave plate is } \\
\text { inserted into the beam } \\
\text { path to produce circular } \\
\text { polarisation. }\end{array}$ & $\begin{array}{l}\text { The polarisation } \\
\text { direction was maintained } \\
\text { at } p^{-} \text {. }\end{array}$ & $\begin{array}{l}\text { The polarisation } \\
\text { direction was maintained } \\
\text { at } s^{-} \text {. }\end{array}$ \\
\hline $\begin{array}{l}\text { Optical } \\
\text { micrographs of } \\
\text { the holes exit } \\
\text { aperture }\end{array}$ & $\overline{300 \mu \mathrm{m}}$ & 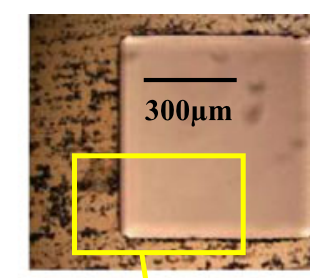 & 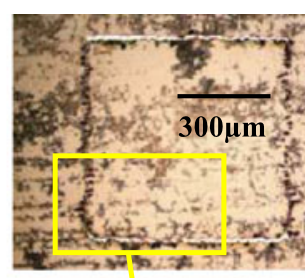 \\
\hline $\begin{array}{l}\text { Description of } \\
\text { the results }\end{array}$ & $\begin{array}{l}\text { The central cut-out was } \\
\text { successfully removed } \\
\text { after a } 500 \text { s laser } \\
\text { exposure. Significant } \\
\text { distortions are present } \\
\text { on the exit side of the } \\
\text { cut-out. }\end{array}$ & $\begin{array}{l}\text { The central cut-out was } \\
\text { successfully removed } \\
\text { after a } 500 \text { s laser } \\
\text { exposure. Minor } \\
\text { distortions are present } \\
\text { on the exit side of the } \\
\text { cut-out. }\end{array}$ & $\begin{array}{l}\text { The central cut-out } \\
\text { could not be removed } \\
\text { after a } 500 \mathrm{~s} \text { laser } \\
\text { exposure. Significant } \\
\text { distortions are visible } \\
\text { along the exit channels. }\end{array}$ \\
\hline
\end{tabular}

The cutting process was successful with both circular and $p$-polarisation, where the central cut-outs were removed by the ultrasonic waves. The optical micrographs in Table 4 show the exit holes after the central square cut-outs have been removed. $s$-polarisation failed to cut out the central square, demonstrating a poorer process efficiency in that case. The optical micrographs in Table 4 show the exit grooves produced with $s$-polarisation, with the central cutout still in place. When the test was repeated with a $10 \%$ shorter processing time, only the $p$-polarisation successfully cut out the central square, suggesting this polarisation mode provides a further improvement in cutting efficiency compared with circular polarisation under these experimental conditions. Of these two configurations, microscopic investigation revealed that the surface quality of the sidewalls was better with $p$-polarisation (Fig. 7), whereas circular polarisation produced ripples and distortions on the sidewalls of the hole (Fig. 8). Figures $7 \mathrm{~b}$ and $8 \mathrm{~b}$ show the amplitude of these ripples on selected regions of the sidewalls for $p$ - and circular polarisation, respectively, measured with an optical surface profiling system (WYKO NT1100). The average peak-to-peak amplitude between the valleys (in dark blue) and the peaks (in light red) was found to be $2.5 \pm 1 \mu \mathrm{m}$ for $p$ - and $5 \pm 1 \mu \mathrm{m}$ for circular polarisation.
The better process efficiency and quality obtained with $p$-polarisation can be explained by the increased coupling of the laser beam energy to the sidewalls during the machining of the micro-channels, compared with circular or $s$-polarisation. For a typical high aspect-ratio channel geometry, the angle of incidence on the walls is around $80^{\circ}$. The corresponding value for the reflectivity of steel is around $47 \%$ for $p$-polarisation and $94 \%$ for $s$-polarisation [10]. Therefore, the intensity of the light reflected from the walls is approximately twice as much with $s$-polarisation compared with $p$-polarisation. This results in a higher loss of energy through the exit of the channels machined with $s$ polarisation. The extra reflections on the non-optically flat walls inside the channels also induce a non uniform energy distribution resulting in distorted exit channels produced with $s$-polarisation. In the case of circular polarisation, the $p$ - and $s$-polarisations are averaged over time. This produces an average value for reflectivity which results in an intermediate quality and efficiency for micromachining.

\section{Conclusions}

A method for dynamically switching the polarisation direction of a femtosecond laser beam with a fast-response, trans- 


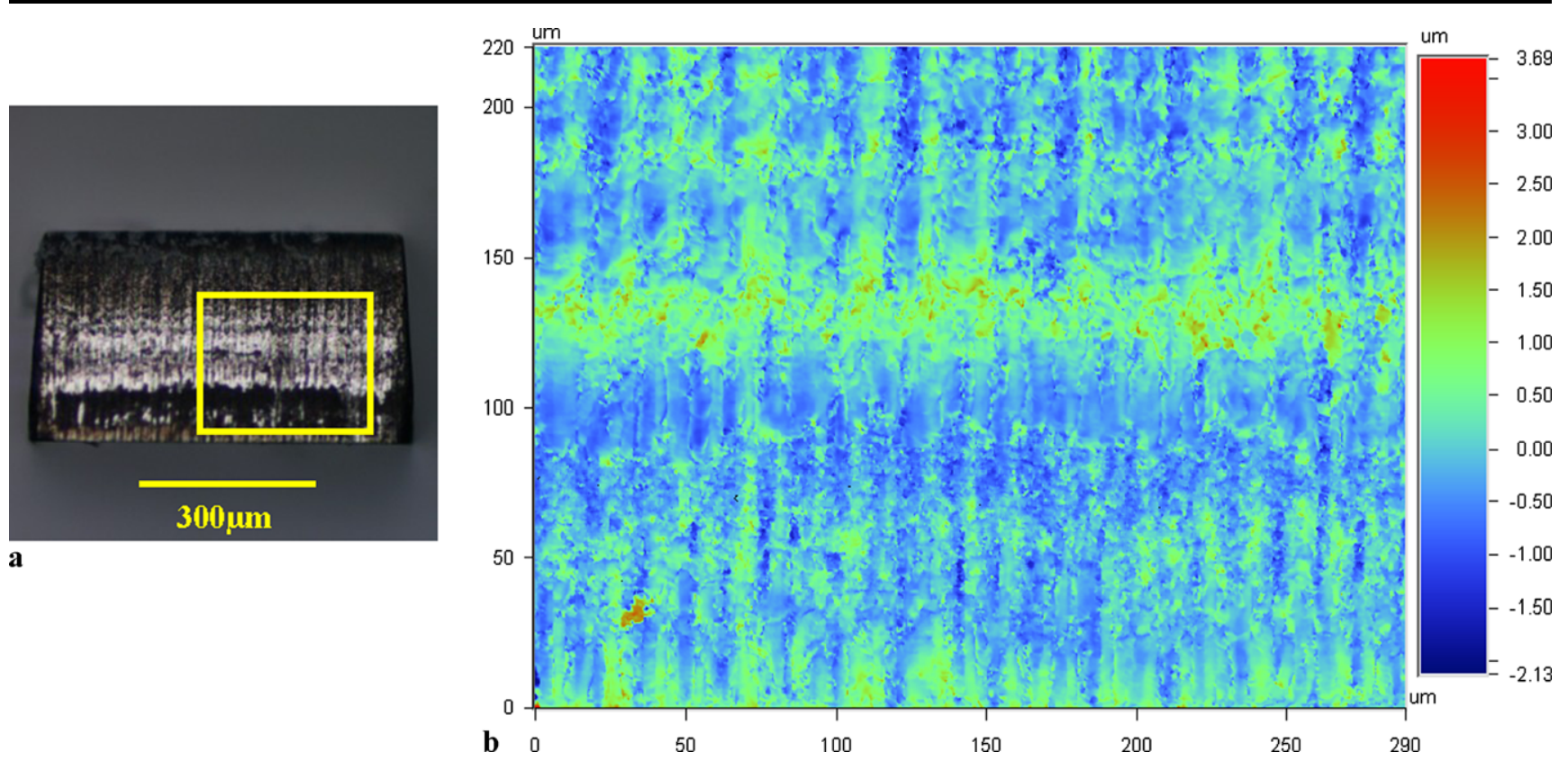

Fig. 7 (a) Optical micrograph of a square cut-out with $p$-polarisation after removal by ultrasonic waves (see Table 4). The sidewall of the cut-out is shown here, with magnified area (yellow square). (b) Magnified area observed with an optical surface profiling system, showing vertical periodic ripples on the sidewall. The dark blue regions are the valleys and the light orange regions are the peaks. The colour-coded scale shows the distance from an average mid-plane, in $\mu \mathrm{m}$

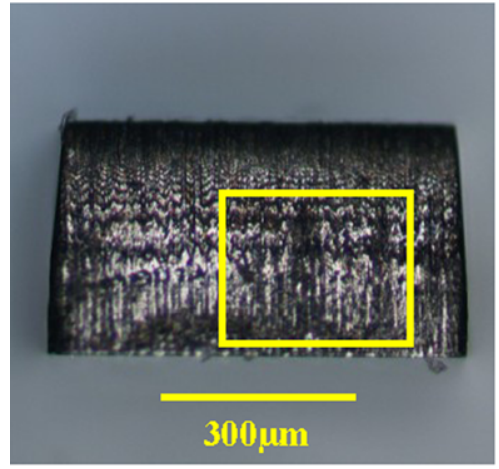

a

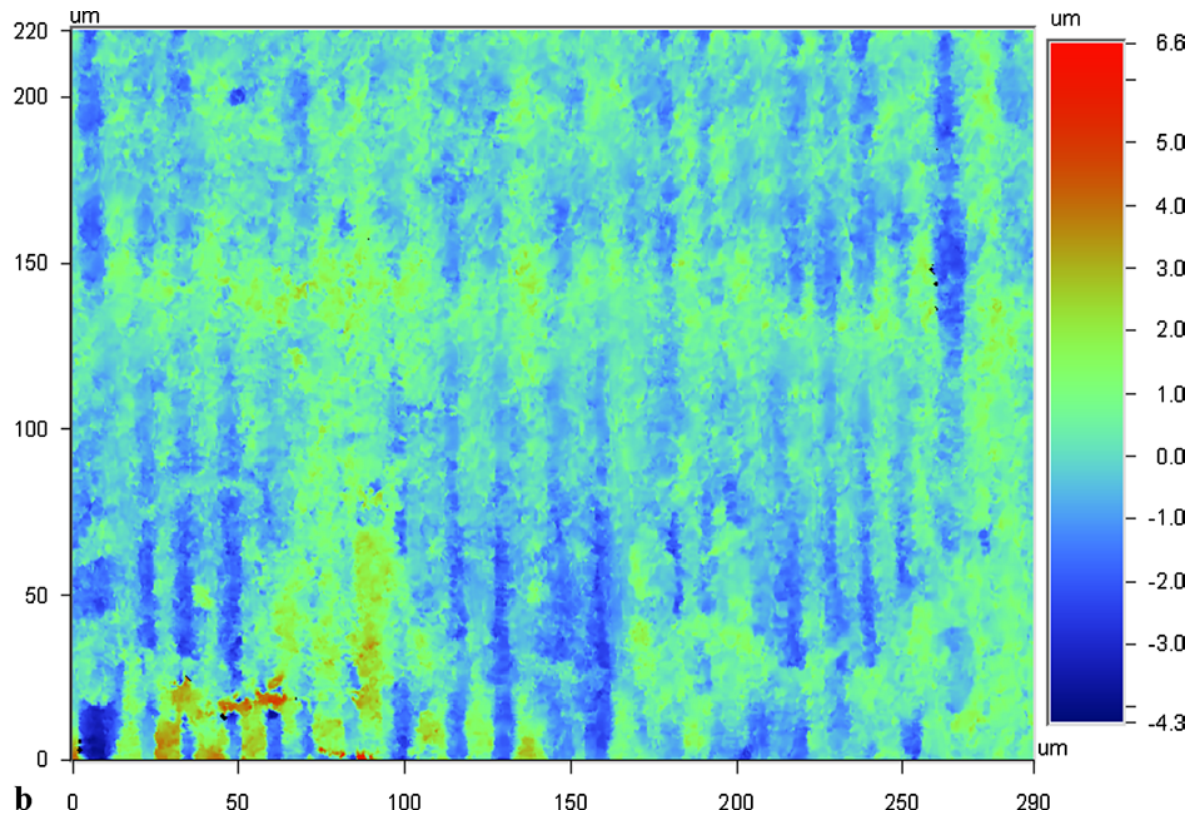

Fig. 8 (a) Optical micrograph of a square cut-out with circular polarisation after removal by ultrasonic waves (see Table 4). The sidewall of the cut-out is shown here, with magnified area (yellow square). (b) Magnified area observed with an optical surface profiling system,

missive, ferroelectric liquid-crystal device has been presented. Helical drilling and cutting of high-aspect-ratio microscopic structures in a steel plate was tested, using various polarisation driving modes and testing different drilling ge- showing vertical periodic ripples on the sidewall. The dark blue regions are the valleys and the light orange regions are the peaks. The colour-coded scale shows the distance from an average mid-plane, in $\mu \mathrm{m}$ ometries. Microscopic investigation of the resulting features revealed that polarisation trepanning with a liquid-crystal device offers better levels of machining quality than circular or static linear polarisations. 
To our knowledge, this is the first time polarisation trepanning has been achieved using a solid-state device for micro-processing, providing polarisation rotation without any undesirable mechanical motion, associated vibrational problems and maintenance downtime.

Due to its design characteristics, the liquid-crystal device used in this research had an angular rotation range limited to $90^{\circ}$ and required a DC-balanced, $50 \%$ duty-cycle periodic driving voltage. As a result, the tests were restricted to machining axi-symmetric features. Future work will aim to assess, amongst the wide range of liquid-crystal technologies available, the most suitable ones for various drilling and machining configurations.

Although the machining quality often associated with high-precision trepanning optics has not been achieved, the fast-response liquid-crystal polarisation rotator presented here could emerge as a powerful alternative. As an off-theshelf item, it can easily provide improved machining quality for existing laser systems.

Acknowledgements The authors gratefully acknowledge the support of the EPSRC and the help of Mr. Kipp Bauchert, Boulder Nonlinear Systems, Inc., who provided the liquid-crystal polarisation rotator used in this research as well as that of Dr. Mary Erlund for her editorial contribution.

\section{References}

1. D. Breitling, C. Föhl, F. Dausinger, T. Kononenko, V. Konov, Top. Appl. Phys. 96, 131-156 (2004)
2. F. Dausinger, Proc. SPIE 5777, 840-845 (2005)

3. D. Breitling, A. Ruf, F. Dausinger, Proc. SPIE 5339, 49-63 (2004)

4. N. Sudani, K. Venkatakrishnan, B. Tan, Opt. Lasers Eng. 47, 850854 (2009)

5. N. Rizvi, RIKEN Rev. 50, 107-112 (2003)

6. A. Ostendorf, T. Bauer, F. Korte, J.R. Howorth, C. Momma, N.H. Rizvi, F. Saviot, F. Salin, Proc. SPIE 4633, 128-135 (2002)

7. P.S. Banks, M.D. Feit, A.M. Rubenchik, B.C. Stuart, M.D. Perry, Appl. Phys. A 69, S377-S380 (1999)

8. S. Hahne, B.F. Johnston, M.J. Withford, Appl. Opt. 46, 954-958 (2007)

9. C. Föhl, F. Dausinger, Proc. SPIE 5063, 346-351 (2003)

10. S. Nolte, C. Momma, G. Kamlage, A. Ostendorf, C. Fallnich, F. von Alvensleben, H. Welling, Appl. Phys. A 68, 563-567 (1999)

11. C. Föhl, D. Breitling, F. Dausinger, Proc. SPIE 5121, 271-279 (2003)

12. H.K. Tönshoff, C. Momma, A. Ostendorf, S. Nolte, G. Kamlage, J. Laser Appl. 12, 23-27 (2000)

13. V.G. Niziev, A.V. Nesterov, J. Phys. D, Appl. Phys. 32, 1455-1461 (1999)

14. F. Dausinger, J. Shen, ISIJ Int. 33(9), 925-933 (1993)

15. F. Dausinger, RIKEN Rev. 50, 77-82 (2003)

16. Z. Kuang, D. Liu, W. Perrie, S. Edwardson, M. Sharp, E. Fearon, G. Dearden, K. Watkins, Appl. Surf. Sci. 2009.02.043 (2009)

17. L. Mellor, S. Edwardson, W. Perrie, G. Dearden, K. Watkins, Proc. ICALEO 2009 N105, pp. 1329-1337 (2009)

18. Z. Guosheng, P.M. Fauchet, A.E. Siegman, Phys. Rev. B 26, 5366$5381(1982)$

19. I. Ursu, I.N. Mihăilescu, A.M. Prokhorov, V.N. Tokarev, V.I. Konov, J. Appl. Phys. 61, 2445-2457 (1987)

20. L. Nanai, R. Vajtai, I. Hevesi, R. Laiho, L. Heikkila, Superlattices Microstruct. 11, 435-438 (1992)

21. P. Mannion, J. Magee, E. Coyne, G.M. O'Connor, Proc. of SPIE, vol. 4876 (2002) 